\title{
Therapy of a Case Studyof Rheumatoid Arthritisby Iranian Traditional Medicine
}

\author{
SeyyedmohammadBagher Fazljou ${ }^{1}$, SolmazRahmani Barouji ${ }^{2}$, ParinazRahmani Barouji ${ }^{3^{*}}$ \\ ${ }^{1}$ Assistant Professor. PhD of Iranian Traditional Medicine, Head of Traditional Medicine, Tabriz University of \\ Medical Sciences, Tabriz, Iran. \\ ${ }^{2}$ PhD student of Iranian Traditional Medicine, Tabriz University of Medical Sciences, Tabriz, Iran. \\ ${ }^{3}$ M.s Industrial Management, AllameTabataba`e University, Tabriz, Iran.
}

*Corresponding Author: ParinazRahmaniBarouji, M.s Industrial Management, AllameTabataba`e University, Tabriz, Iran.

\section{Abstract:}

Objective: We hope to report this case with rheumatoid arthritis with other Iranian Traditional medical treatments for treating this disease with other methods of both Iranian traditional medicine and Modern Medicine or the combinations of methods.

Background and Aim: Rheumatoid arthritis is one of the causes of motor constraints and articular problems. The most important symptoms are motor limitation and small joints, which is an immunological disease and is an antibody in the body. And diagnostic laboratory tests are variable in this disease:
1. High ESR
2. Positive ANA
3. Positive Anti CCP.

There is no definitive treatment in modern medicine for this disease, but only by suppressing the immune system through the administration of a variety of corticosteroids is partly prevented from flares. This paper presents a case study of a patient with rheumatoid arthritis with an Iranian Traditional medicine approach (without the use of modern medicine).

Case Present: A 47-year-old patient (A man) was diagnosed with rheumatoid arthritis, treated with lifestyle modification and drugs and allergic catarrh according to the knowledge of Iranian Traditional medicine.

Results: It seems that the symptoms of the disease are very similar to the allergic (acute or chronic) symptoms that Iranian remedies have been treated with lifestyle modification, cleansing and control of pain and strengthening of the joints, as well as moderating the power of healing (GovvehyeModabbereh), without the need to use Classical medicine

Keywords: Rheumatoid Arthritis, Catarrh, Acute or chronic allergic catarrh, Iranian Traditional medicine, Joints pain

\section{INTRODUCTION}

Rheumatoid arthritisis the most common anti - inflammatory disease in the joints and is among the autoimmunediseases that create significant inability to infected people. [5]The criteria for detecting the conflict is at least one joint with inflation and pain.in regard to what has become known in the Iranian Traditional medicine as temperamentwhich is considered as a spectrum. Any way out of this spectrum is treated as Dystemperamentor disease.Being a catarrhallergicreaction in the patient, the pain and swelling of the wrist and the wristjoints, and the ankles with the onset of the morning, and the swelling of symptoms in the cold weather, the gravity of the joints, and seeing the dreams of snow and rain, are the Symptomsof phlegmatic catarrh allergic. Rheumatoid arthritis is a chronic inflammatory joint disease, which can cause cartilage and bone damage as well as disability. Early diagnosis is key to optimal therapeutic success, particularly in patients with well-characterized risk factors for poor outcomes such as high disease activity, presence of autoantibodies, and early joint damage. [1] 
Rheumatoid arthritis is characterized by persistent synovitis, systemic inflammation, and autoantibodies. 50\% of the risk for development of rheumatoid arthritis is attributable to genetic factors. Smoking is the main environmental risk. In industrialized countries, rheumatoid arthritis affects $0 \cdot 5-1 \cdot 0 \%$ of adults, with 5-50 per 100000 new cases annually. The disorder is most typical in women and elderly people. Uncontrolled active rheumatoid arthritis causes joint damage, disability, decreased quality of life, and cardiovascular and other comorbidities [2]

In rheumatoid arthritis,synoviocytes and macrophages produce joint-damaging cytokines. These pro-inflammatory cytokines inhibit the synthesis of proteoglycans and collagen and increase their degradation. [18]In traditional Persian literature, the term rheumatoid arthritis is expressed as a disturbance in humoral balance of the body, which commonly occurs in subjects with 'cold' and 'moist' temperaments. Patients with this humoral disorder suffer from chronic pain in the joints 'waja'-e-mafasel', mainly due to accumulation of phlegm in the joints. $(19,20)$ Various medicinal plants are traditionally used for the management of symptomatologies associated with RA in Persian medicine.

In this article, we describe a rheumatoid arthritis case that has been treated with the Iranian Traditional medicine.

\section{IRANIAN TRADITIONAL MEDICINE}

As defined by the World Health Organization, Traditional Medicine is a collection of knowledge, skills and actions based on the theories, beliefs and experiences of indigenous peoples of different cultures, whether or not they can be described, in the health, prevention, diagnosis, improvement and treatment of physical illnesses and mentally used. [12] Iranian Traditional Medicine dates back almost 3,000 years ago. Studies and researches reveal that some of the earliest records of history of ancient Iranian medicine can be found in 8,000 to 6,500 B.C. [3] while it is remarkable that Hippocrates, a Greek physician who is considered as one of the outstanding figures in the history of medicine belongs to about $460 \mathrm{BC}$.[3] Iranian traditional medicine is known as a holistic medicine emphasizes on individual differences, healthy lifestyle. [4]. The life style rules in Iranian traditional medicine are mainly focused on six core principles, including food and drinks, climatic conditions and environment, sleeping and waking, physical activities, psychiatric conditions and ridding body from waste materials (sweat, urine or feces). One of the most significant differentiation sides of Iranian Traditional Medicine school compared to other medical schools is essential role of health prevention and preservation, so the medicine has been described by Avicenna, a great ancient Iranian scientist, as the following comprehensive definition: "The health maintenance when you are healthy, and its restoration when you are sick." [11] Iranian Traditional Medicine focuses on each person in order to find the best treatment due to different physical andmental characteristics of patient that can be understood. [8] Everybody has a definite Mizaj which is determinant to construct all physical or mental characteristics. Mizaj is a quality concluded of compounding every four basic elements (Arkan) with each other in a specific proportion and their interaction. These basic elements are: Water, fire, soil, and wind, producing kinetic energy (warmness) and fluidity (humidity) in the body.

Each element has a special quality: air is hot and wet, water is cold and wet, soil is cold and dry, and fire is hot and dry. The mixing of these four elements can cause different temperaments. $[6,16]$ Human health depends on the maintenance of the Mizaj in a balanced state. Every mal-temperament or Sou-e-mizaj could lead to illness. [12,13,14,15] Iranian Traditional Medicine tries to propose a proposal to increase the effectiveness of therapeutic approaches along with reducing side effects by choosing the appropriate protection and health principles. [4] When normal life style modifications are not capable of treating an illness, the Iranian traditional medicine offers a massive list of herbal medicine, including over 2,500 types of medicinal plants, or applies a variety of non-chemical methods such as bloodletting, massage, thermotherapy, hydrotherapy, etc. for different types of diseases. Since the majority of methods and drugs applied in the Iranian traditional medicine have very insignificant side effects and health risks and, at the same time, they result in more sustainable and long-lasting health benefits, the medical tradition is becoming increasingly popular in Iran and many other parts of the world. [17]

\section{Patient Introduction}

Patient(aman) 47 years old with catarrh allergic symptoms, pain and swelling of the wrist and wrist joints, and ankles pain that intensified with the onset of the morning, the symptoms intensify in the 
cold weather and healed in the hot weather,there was a feeling of gravity in joints of patient by cutting off the Corticosteroid treatment, Symptoms had been returned.

- In this case,diagnostic laboratory tests were as fallow:

1. Uric Acid = 12 (normal up to 8)

2. High ESR (ESR $1 \mathrm{~h}=89)$

3. Positive CRP

4. Positive ANA

5. Positive Anti CCP

The patient has referred to the clinic of IranianTraditional Medicine with thesymptoms were shown above.

\section{THERAPY METHOD}

- At the first referral for this patient with phlegmatic catarrh allergic symptoms, treatment was first started by Lifestyle modification to adjust and regulate power of healing (GovvehyeModabbereh )withthe steps described as follows:

1. Sleeping in time

2. Regular exercises

3. Avoiding cold snacks such as dough and yogurt, tomatoes and cucumbers

4. Good chewing,

5. Eating slowly

6. Avoiding over - eating

7. Not drinking liquids along with Food and drinking at least an hour after food

8. Avoiding raw fruits

9. Using sheep woolen mattress for sleeping

- As a medicine: anti gout decoction as moderrnegres

1. Monzij Phlegm

2. Laxative Phlegm

3. These two itemspointed above, Improve the catarrhwhich considered in Iranian Traditional medicine as the main cause of joint disease.

4. Davayebalgham(PhlegmDrug)

5. Razicapsule for joints

6. Stachys ( StachysLavandulifolia ) were prescribed.

7. Allergic cupping( Hijamat ) has been prescribed for the patient to moderate the power of healing ( GovvehyeModabbereh )

- In the Second Visit

1. The problems of the hands were greatly resolved and the leg problems were mild.

2. Continuation of the previous orders and the oil therapy was donated with bitter almond oil and rehabilitation ointment(PomadeTavanbakhshi )

3. Edible alfalfa oil was given three times a day for the patient.

- In theThird Visit

1. The recovery is apparent at the same trend. Treatment continued with Colchicum speciosumcapsule BID ( Bis In Die )

2. Continuing the previous orders

- In the Fourth Visit

The patient was advised to use orders below in order to improve power and energy of his body:

1. Almond

2. Sheep stock

3. Oleaster(Elaeagnusangustifolia,sinjid) flour 
4. Stachys( StachysLavandulifolia )

5. Davayebalgham(PhlegmDrug)

6. Edible alfalfa oil

And if there wasany pain, using the Razi capsule for joints pain had been prescribed

The patient was controlled every 6 months, and the patient stated that he did not need any medicine by respecting healthy lifestyle and he had been recovered completely.

Edible Ingredients Combination prescribed for the patient were as follows:

Table1.Edible Ingredients Combination prescribed for the patient

\begin{tabular}{|c|c|}
\hline Name of the drug & Ingredient \\
\hline Monzij Phlegm & $\begin{array}{ll}- & \text { PimpinellaAnisum } \\
- & \text { Melissa Officinalis } \\
- & \text { Adiantumcapillus-veneris } \\
\text { - } & \text { Ficuscarica } \\
\end{array}$ \\
\hline Laxative Phlegm & $\begin{array}{ll}\bullet & \text { Rosa Damascena } \\
- & \text { Senna Alexandrina } \\
\end{array}$ \\
\hline Davayebalgham ( Phlegm Drug ) & $\begin{array}{ll}- & \text { ZatariaMultiflura } \\
\text { - } & \text { Nigella Sativa } \\
\text { - } & \text { Pistacialentiscus } \\
\text { - } & \text { TrachyspermumAmmi } \\
\text { - } & \text { Honey }\end{array}$ \\
\hline Razi capsule for joints pain & $\begin{array}{ll}- & \text { Aloe Barbadensis Miller } \\
- & \text { CarumCarvi(Caraway) } \\
\text { - } & \text { ZingiberOfficinale } \\
\text { - } & \text { Colchicum speciosum } \\
\end{array}$ \\
\hline Colchicumspeciosum capsule & $\begin{array}{ll} & \text { Colchicum speciosum } \\
\end{array}$ \\
\hline Rehabilitation ointment(Pomade Tavanbakhshi) & $\begin{array}{ll}- & \text { Camels Hump } \\
- & \text { PrunusAmygdalusVar Amara (Bitter Almond Oil) } \\
- & \text { Medicago Sativa }\end{array}$ \\
\hline
\end{tabular}

\section{ORDERS AND EFFECT OF THEM}

The orders of the drugs mentioned in Table 1 and effect of them were as follows:

\section{MonzijPhlegm:}

Should be taken Every morning, before breakfast, in boiled water for 10 days

\section{Laxative Phlegm:}

Laxative is taken on 11th dayto exorcise phlegm.

\section{Davayebalgham(PhlegmDrug):}

Be taken as much as a hazelnut with lukewarm water 3 times a day to exorcise phlegmin the body organs and melt them and warm the body.

\section{Razi capsule for joints pain:}

BID ( Bis In Die ) for joint pain

\section{Colchicumspeciosum capsule:}

Be taken 3 times a day for removing joint edema, arthritis and gout

\section{Rehabilitation ointment(PomadeTavanbakhshi ):}

Used 3 times a day topically

\section{Results}

In the last visit, the course of recovery was increased and the pain was controlled. The problems of the hands were greatly resolved and the leg problems were mild. The response to the test was in the normal range and after the discontinuation of the drug did not have any symptoms of relapse.Diagnostic laboratory tests were as follow:

- Uric Acid = 8 (Normal)

- $\quad \mathrm{ESR}=$ Normal

- $\quad \mathrm{CRP}=$ Negative 
It seems that the symptoms of the disease are very similar to the allergic (acute or chronic) symptoms that Iranian remedies have been treated with lifestyle modification, cleansing and control of pain and strengthening of the joints, as well as moderating the power of healing (GovvehyeModabbereh ), without the need to use Classical medicine. During the monthly and multiple follow-up, gradually the symptoms of the patient were reduced and the results were satisfactory. The response to the test was in the normal range and after the discontinuation of the drug did not have any symptoms of relapse. Diagnostic laboratory tests showed a successful treatment trend.

\section{DISCUSSION}

As seen, the symptoms of this patient include: the presence of allergic discomfort, pain and swelling of the wrists and joints, and the ankles with the onset of the morning and exacerbation of symptoms in the cold, the gravity of the joints and seeing the dreams of the snow and rain of the patient are very similar to the symptoms of overcoming melancholy and, in terms of Perspective of Iranian Traditional medicine. Then the treatment was taken phlegm mal-temperament (Soo-e-mizaj- balgham). In addition, the patient's regimen was adjusted with allergic hygiene. This paper attempts to express a case report on rheumatoid arthritis that has been treated by Iranian traditional medicine. Given the effectiveness of treatment with Iranian traditional medicine, in some cases, the focus on traditional medicine by itself can`t be effective. In cases of deformed joints in rheumatoid arthritis and joints deformity, joints repair is difficult, but if deformity of the joints is low, traditional therapies such as cupping, dry cupping, leaching therapy, can be used. In patients with rheumatoid arthritis, GovvehyeModabbereh should be kept moderateneither more nor less. We can also massage the body with warm oils, such as chamomile oil, blackened grains, Because the nature of the joints is cold in these patients, and the phlegm in the joints has fallen and accumulated, we try to keep the joints as warm as possible.

In addition to massage therapy, we can also strength the joints, muscles and nerves. Eating edible alfalfa and alfalfa oil and alfalfa sweeteners is beneficial. In patients with rheumatoid arthritis, the body should be kept as warm as possible, avoided by cold weather and avoided by cold foods. In order to control stress and nervous pressure in these patients, one should try to maintain the best possible lifestyle.

\section{CONCLUSION}

This paper attempts to express a case report on rheumatoid arthritis that has been treated by Iranian traditional medicine.This case report showed that rheumatoid arthritis can be treated with medicine. As seen, the symptoms of this patient are very similar to the symptoms of overcoming melancholy and, in terms of Perspective of Iranian Traditional medicine. Then the treatment was taken phlegm mal-temperament (Soo-e-mizaj- balgham) . In addition, the patient's regimen was adjusted with allergic hygiene. During the monthly and multiple follow-up, gradually the symptoms of the patient were reduced and the results were satisfactory. The response to the test was in the normal range and after the discontinuation of the drug did not have any symptoms of relapse. Diagnostic laboratory tests showed a successful treatment trend.

Highlight: Rheumatoid ArthritisDisease can be treated by therapies through Iranian Traditional Medicine and diagnostic laboratory tests can be recovered and subsided to the normal level.

\section{REFERENCES}

[1] Smolen, Josef S.; Aletaha, Daniel; McInnes, Iain B. (2016-10-22). "Rheumatoid arthritis". Lancet. 388 (10055): 2023-2038

[2] Scott DL,Wolfe F, Huizinga TW,Rheumatoid arthritis,Lacent, 2010 Sep 25;376(9746):1094-108.

[3] Mirghazanfari, Seyyed Mahdi (2017-05-24), Iranian Traditional Medicine prioritizes health maintenance over treatment, Tehran Times. Retrieved 2018-05-14.

[4] Nejatbakhsh, F.; Siahpoosh, M.; 2014, Iranian Traditional Medicine Handbook: Diagnosis and Treatment of Diseases, Tehran Publication, Tehran, Iran.

[5] Guyton, Arthur; Textbook of medical physiology, 10th ed. 2000, Translated by Alirezaei, Ali, Arjmand publication, Tehran, Iran 
[6] AghiliKhorasani, Hakim Mohammad Hussein. Makhzan al advieh, First Publication, Tehran University of Medical Sciences (2008), Tehran, Iran

[7] Hakim A'zamkhan, Muhammad. EksirA'zam. Fourth volume, Volume II, Iran University of Medical Sciences, Institute of History of Medicine, Islamic Medicine and Supplement (2008).

[8] Rezaeizadeh H, Alizadeh M, Naseri M, Ardakani MS. The traditional Iranian medicine point of view on health and disease. Iranian Journal of Public Health. 2009; 38(Suppl. 1): 169-172.

[9] ValiolahMozafarian, Recognition of Iranian medicinal and aromatic herbal, FarhangMoaaser Publication, 2017, Tehran, Iran.

[10] Harrison's Rheumatology 4th Ed, 16 Dec 2016, Anthony S. Fauci; Carol A. Langford, McGraw- Hill Education,publication; OH, United States

[11] Hatami H. Public health and preventive medicine according to Avicenna. Sci J Islam Repub Iran Med Counc. 2000; 18:223-38.

[12] Naseri M, Rezayizadeh H, Choupani R, Anoushirvani M. 10th ed. Tehran: Iranian Traditional Medicine Publication; 2013. A Review on Iranian Traditional Medicine Principles; p. 1392. (In Persian)

[13] Yousefifard M, Parviz M, Hosseini M, Ebadiani M, Keshavarz M. Mizaj; past, present and future. PhysiolPharmacol. 2013; 16:328-39.

[14] Hatami H. Public health and preventive medicine according to Avicenna. Sci J Islam Repub Iran Med Counc. 2000; 18:223-38. (In Persian)

[15] Akbarzadeh A, Salehi A, Nimrouzi M. Preventive medicine in view of Hakim Jorjani. J Med Hist. 2012; 13:39-54. (In Persian)

[16] Jackson WA. A short guide to humoral medicine. Trends Pharmacol Sci. 2001; 22(9): 487-9

[17] Press TV, Iranian traditional medicine key to optimal health, life style; Wed Jun 20, 2018 02:22PM [Updated: Thu Jun 21, 2018 11:11AM]

[18] Mohammad HoseinFarzaei, FatemehFarzaei, Mohammad Abdollahi,

[19] Zahra Abbasabadi, Amir Hossein Abdolghaffari, Bahman Mehraban Amechanistic review on medicinal plants used for rheumatoid arthritis intraditional Persian medicine, J Pharm Pharmacol. 2016 Oct;68(10):1233-48.

[20] Avicenna. Canon of Medicine. New Delhi, India: S WarisAwab, Lamia Hamdard Printing Press, 1998.

[21] Jorjani SE. ZhakhirehKharazmshahi. Tehran, Iran: Iranian Cultural Organization Press, 1976.

Citation: ParinazRahmaniBarouji, et al., (2019). Therapy of a Case Study of Rheumatoid Arthritis by Iranian Traditional Medicine. International Journal of Medicinal Plants and Natural Products (IJMPNP), 5(3), pp.23-28.http://dx.doi.org/10.20431/2454-7999.0503004

Copyright:(C) 2019Authors, this is an open-access article distributed under the terms of the Creative Commons Attribution License, which permits unrestricted use, distribution, and reproduction in any medium, provided the original author and source are credited. 